\title{
Développement et validation d'une méthode quantitative de dosage par Chromatographie Liquide à Haute Performance Ultra-violet (CLHP-UV) de l'artémisinine dans Artemisia annua cultivé au Bénin
}

\author{
Hippolyte GANSE ${ }^{1 *}$, Fernand GBAGUIDI ${ }^{1}$, Taofiki AMINOU ${ }^{3}$, Hermine ZIME ${ }^{1}$, \\ Mansourou MOUDACHIROU ${ }^{1}$ et Joëlle QUETIN-LECLERCQ ${ }^{2}$ \\ ${ }^{1}$ Laboratoire National de Pharmacognosie /CBRST Porto-Novo (Bénin) 01 BP 06. \\ E-mail : senakponganse@yahoo.fr,zimegani@yahoo.fr,ahokannou@yahoo.fr,moudmans@yahoo.fr \\ ${ }^{2}$ Laboratoire de Pharmacognosie, Unité CHAM, Département de Pharmacie, Université Catholique de \\ Louvain UCL 7230 Av. E.Mounier.72.1200Bruxelle, Belgique.E-mail : joelle.leclercq@uclouvain.be. \\ ${ }^{2}$ Laboratoire d'Expertise et de Recherche en Chimie de l'Environnement et de l'Eau (LEERCE) Université \\ d'Abomey Calavi 01BP 526 aminoutaofiki@yahoo.fr. \\ *Auteur correspondant ; E-mail : senakponganse@yahoo.fr; Tèl : + (229) 90030452
}

\section{RESUME}

Artemisia апnиa, espèce de la famille des asteraceae est une plante dont les feuilles contiennent une lactone sesquiterpénique appelée artémisinine qui est efficace contre toutes les souches de parasites responsables du paludisme. Cette étude nous a permis de développer et de valider une méthode de Chromatographie : la méthode de Chromatographie Liquide à Haute Performance (CLHP), dans le but de quantifier l'artémisinine dans les poudres de feuilles d'Artemisia annua cultivé au Bénin. La méthode de Chromatographie Liquide à Haute Performance développée, est validée pour les quantités en artémisinine de 200 à $1200 \mathrm{ng}$. Les analyses fournissent une bonne : exactitude, reproductibilité, et sélectivité pour l'analyse quantitative de l'artémisinine. La courbe de calibration est linéaire avec un coefficient de corrélation $\mathrm{R}^{2}=$ 0,9988. Les coefficients de variations $(\mathrm{CV})$ intra-jour ou répétabilité et inter-jours ou fidélité intermédiaire sont inférieurs à $5 \%$ pour toutes les séries de quantités en artémisinine. Les taux de récupération de l'artémisinine par la méthode d'extraction au soxhlet sont d'environ 98\%. Les limites de détection et de quantification ont été déterminées : elles sont respectivement inférieures à 2,4 et $8 \mathrm{ng}$. La teneur en artémisinine est d'environ $0,3 \%$ pour la poudre de feuilles d'artemisia annua cultivé au Bénin.

(C) 2011 International Formulae Group. All rights reserved.

Mots clés : CLHP-UV, artémisinine, artemisia annua.

\section{INTRODUCTION}

La malaria est depuis longtemps connue comme un des soucis principaux de santé publique en Afrique Sub-saharienne. On estime que 300 à 500 millions de personnes sont atteintes tous les ans de la malaria (Towie, 2006). Les efforts déployés pour lutter contre le paludisme étant contrariés par l'apparition et la propagation de résistances à la plupart des molécules présentes sur le marché (la chloroquine, la quinine, le fansidar, etc...), il en résulte une proportion importante de décès dans les pays en voie de développement (Elisabeh, 2006). Pendant longtemps, les programmes de gestion de la malaria dans les pays endémiques se sont 
fondés sur des traitements monothérapiques. Cependant, sur la base de l'évidence de l'efficacité, l'Organisation Mondiale de la Santé a recommandé l'application des thérapies de combinaisons contenant les dérivés de l'artémisinine (médicaments ACT : Artemisinin-based Combination Therapy) (Davis et al., 2005). Elles sont à ce jour considérées comme les plus efficaces contre le paludisme.

L'artémisinine est une lactone sesquiterpénique extraite des feuilles de la plante chinoise Qinhao (Artemisia annua). Espèce de la famille des Asteraceae, la plante est employée dans la médecine chinoise depuis plus de 1500 ans. Ses feuilles, ses fleurs et ses petites tiges contiennent de l'artémisinine (Figure 1) qui est le principe actif (Hans et al., 2006).

De nombreuses méthodes analytiques ont mis en évidence l'identification et la quantification de l'artémisinine par: la Chromatographie sur Couche Mince (CCM) (Myriam and Simmonet, 2002; Gabriels and Plaizier-Vercammen, 2004; Marchand et al., 2008) ; la Chromatographie sur Couche Mince à Haute Performance avec détection à l'UltraViolet (CCMHP-UV) (Marchese et al., 2001 ; Bhandari et al., 2005); la Chromatographie Liquide à Haute Performance avec détection à l'Ultra-Violet (Rodger et al., 2009; Qian et al., 2005; Jorge and Javier, 2009 ; Celeghini et al., 2006) puis par évaporation diffusion de la lumière (Hu and She, 2006 ; Peng et al., 2007), etc... Notre objectif est de développer et de valider une méthode de dosage de l'artémisinine dans les poudres de feuilles d'Artemisia annua cultivé au Bénin par CLHP-UV. Les critères de validation des procédures analytiques quantitatives de la Commission Société Française des Sciences et Techniques Pharmaceutiques (SFSTP) (Hubert, 2000) ont été utilisés.

\section{MATERIEL ET METHODES Matériel végétal}

Les plantes d'Artemisia annua ont été cultivées dans la ferme de la Faculté des
Sciences Agronomiques (FSA) de l'Université d'Abomey-Calavi.

La plante a été au préalable séchée à la température ambiante dans une chambre à l'abri de la lumière et retournée tous les jours. La plante séchée a été ensuite soigneusement réduite en poudre par un broyeur et stockée à température ambiante (European Pharmacopeia, 2004).

\section{Matériel analytique}

Le système CLHP Merck Hitachi utilisant une colonne Lichrospher RP-18 (5 $\mu \mathrm{m}$; longueur $25 \mathrm{~cm}$, diamètre interne 4,1 $\mathrm{mm}$ ) où règne une température de $30{ }^{\circ} \mathrm{C}$, est composé des modules suivants: une pompe CLHP ; un autosampler; un four pour colonne ; un détecteur à barrettes de diodes ; un ordinateur muni d'un clavier et d'un écran, utilisant pour l'acquisition des données, le logiciel LaChrom Elite. La détection se fait à une longueur d'onde de valeur $260 \mathrm{~nm}$.

\section{Solvants}

Les solvants, en majorité fournis par Sigma Aldrich, étaient de grade analytique pour les extractions, de grade CLHP pour les analyses CLHP.

\section{Méthode de préparation d'extraits}

Une méthode d'extraction a été utilisée (Extraction au soxhlet) : $5 \mathrm{~g}$ de poudre sont utilisés dans $350 \mathrm{~mL}$ d'éther de pétrole pendant 4 heures. Le filtrat obtenu est complété à $320 \mathrm{~mL}$ puis divisé en deux et évaporé séparément. On dissout chaque résidu sec dans $100 \mathrm{~mL}$ d'éthanol.

\section{Méthode d'analyse par CLHP}

La molécule d'artémisinine a été prédérivativée. Son principe consiste à réaliser avant injection une transformation de l'artémisinine non détectable aux UV en $\mathrm{Q}_{260}$ composé stable réagissant aux UV : on dit que l'artémisinine a subi une prédérivatisation (Figure 2). 


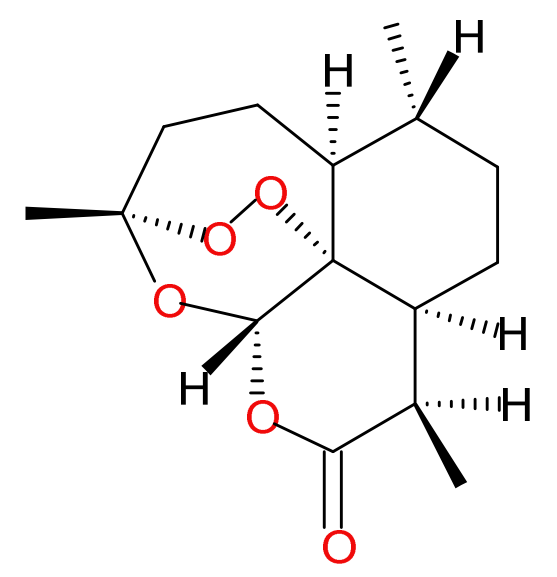

Figure 1 : La structure chimique de l'artémisinine.
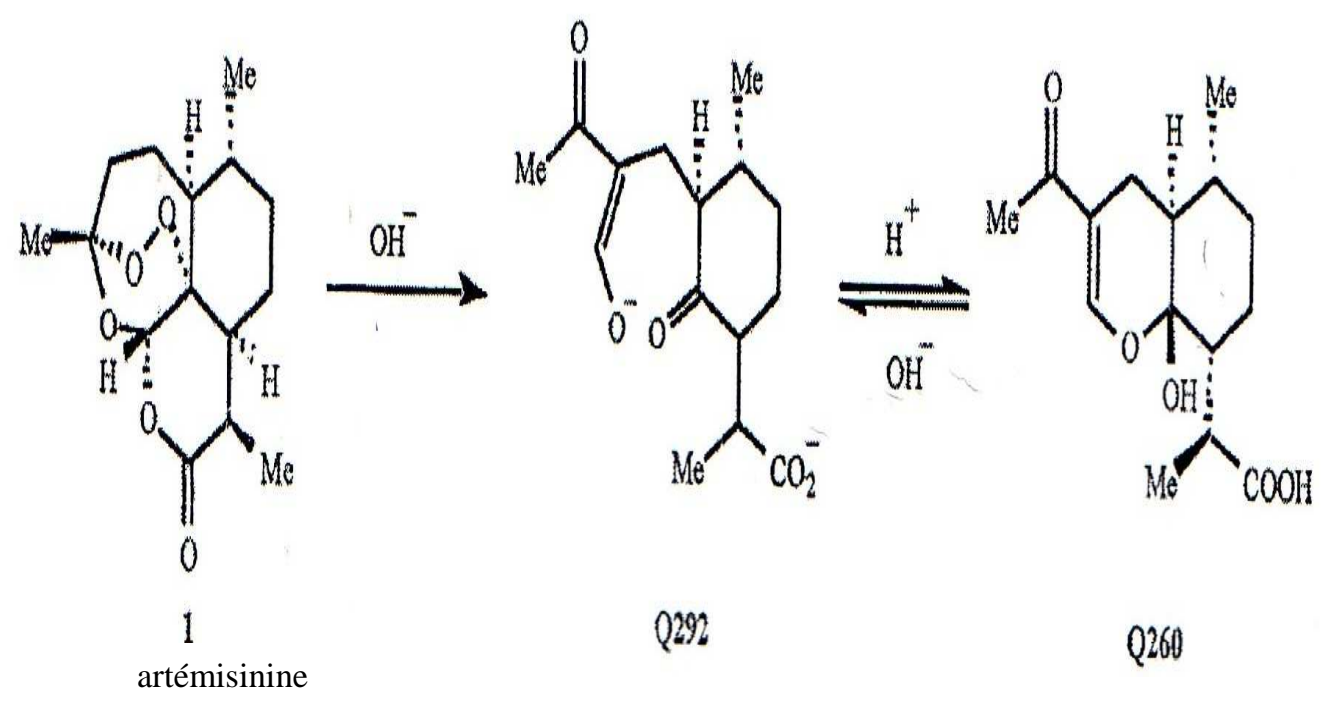

Figure 2 : Equation de la réaction de prédérivatisation de l'artémisinine.

Pour effectuer cette réaction, $1 \mathrm{~mL}$ de solution (extrait éthanolique de poudre de feuilles de Artemisia аппиа ou solution éthanolique d'artémisinine pure) est prélevé dans une fiole jaugée de $10 \mathrm{~mL}$ auquel on ajoute $4 \mathrm{~mL}$ de solution de soude à $0,2 \%$; le mélange obtenu est ensuite porté au bain marie à $50{ }^{\circ} \mathrm{C}$ pendant $30 \mathrm{~min}$; on laisse refroidir pendant 10 min ensuite, on ajoute 1 $\mathrm{mL}$ d'éthanol, puis on complète jusqu'au trait de jauge avec une solution d'acide acétique $0,2 \mathrm{~N}$. Toutes les solutions sont filtrées sur membrane PTFE 0,45 $\mu \mathrm{m}$ avant injection. La phase mobile utilisée est constituée d'un 
tampon phosphate $\mathrm{pH}=6,3$ (solvant $\mathrm{A}$ ) et de méthanol (solvant $\mathrm{B}$ ) respectivement dans les proportions de 55 et $45 \%$. L'analyse est effectuée en mode isocratique avec un débit de $1 \mathrm{~mL} / \mathrm{min}$ et le temps d'analyse est de 15 $\min$.

\section{Procédure de validation et quantification Courbe de calibration}

Pour établir la courbe de calibration, on prélève respectivement $0,1 \mathrm{~mL} ; 0,2 \mathrm{~mL} ; 0,4$ $\mathrm{mL} ; 0,6 \mathrm{~mL}$ de solution mère de $1 \mathrm{mg} / \mathrm{mL}$ (artémisinine pure dans de l'éthanol) soient des concentrations de standards de 10, 20, 40, et $60 \mu \mathrm{g} / \mathrm{mL}$. Le volume de solution injecté étant de $20 \mu \mathrm{L}$, la gamme de quantités en artémisinine est : 200, 400, 800 et $1200 \mathrm{ng}$.

Le modèle de régression est une fonction linéaire $\mathrm{f}(\mathrm{x})=\mathrm{ax}+\mathrm{b}$ avec en abscisse la quantité d'artémisinine introduite et en ordonnée la surface sous courbe du pic correspondant.

\section{Limite de détection et Limite de quantification}

La limite de détection (LD) est la quantité donnant un pic de hauteur égale à trois fois le bruit de fond. La limite de quantification (LQ) est la quantité donnant un pic de hauteur égale à 10 fois le bruit de fond $\mathrm{LD}=3 \mathrm{~s} / \mathrm{a} ; \mathrm{LQ}=10 \mathrm{~s} / \mathrm{a}$ (Hubert, 2006), s=écarttype de la réponse, a=pente de la courbe d'étalonnage.

\section{La répétitivité}

La fidélité intra-jour et inter-jour a été déterminée pour les quatre quantités en artémisinine, trois fois de suite le même jour pour la fidélité intra-jour et trois fois pendant quatre différents jours pour la fidélité interjour.

\section{Taux de récupération}

Le rendement d'extraction a été déterminé à partir de $5 \mathrm{~g}$ de poudre avec ajout de 4,6 mg d'artémisinine.

Taux de récupération $(\%)=(\mathrm{A}-\mathrm{B}) / \mathrm{C} \mathrm{x}$ 100. A est la quantité d'artémisinine extraite du mélange poudre de la plante avec ajout de l'artémisinine. B est la quantité d'artémisinine dans la poudre de la plante sans ajout. C est la quantité d'artémisinine ajoutée.

\section{RESULTATS}

L'artémisinine a été identifié par la méthode CLHP dans les extraits éthanoliques par comparaison des chromatogrammes d'absorption en $\mathrm{Q}_{260}$ du standard et celui de l'échantillon au même temps de rétention 10,697 min (Figure 3).

\section{Critères de validation utilisés Spécificité-Sélectivité}

La méthode développée a permis de séparer et d'établir de manière univoque l'existence de l'artémisinine dans la matrice que représente l'extrait. La qualité de la séparation chromatographique justifie le degré de sélectivité élevé de la procédure de dosage.

\section{Fonction de réponse (courbe de calibration)}

La courbe de calibration a été établie pour une série de quantité de 200 ng à 1200 ng pour la molécule. La méthode a montré une bonne relation linéaire entre les surfaces sous courbes des pics et la série de quantités en artémisinine entre $200 \mathrm{ng}$ et $1200 \mathrm{ng}$.

L'équation de la courbe de calibration de l'analyse de trois ou quatre jours s'écrit: $\mathrm{y}=4,6636 \mathrm{x}-135,89$ avec $\mathrm{R}^{2}=0,9988$.

\section{Linéarité}

Dans l'intervalle de dosage : 200 ng-1200 ng, la réponse (signal) est proportionnelle à la quantité en artémisinine dans l'échantillon: ce qui montre la linéarité de la procédure analytique utilisée.

\section{Fidélité (répétabilité et fidélité intermédiaire)}

Afin d'estimer la fidélité de la technique, les coefficients de variations $(\mathrm{CV})$, de répétabilité (fidélité intra-jour) et la fidélité inter-jours (Tableau 1), ont été déterminés à chaque niveau de quantités en artémisinine.

\section{Limites de détection et de quantification}

La détermination de ces limites conduit au Tableau 2. Ces résultats confirment la linéarité de la méthode développée à l'intérieur de l'intervalle de dosage. 


\section{Taux de récupération}

Les taux de récupération de l'artémisinine par la méthode d'extraction sont d'environ 98\%: ce qui est excellent, compte tenu de la complexité de la matrice végétale ; résultats intéressants par rapport aux travaux de Simmonet et al. (91\%).

\section{Teneur en artémisinine}

L'équation de la courbe de calibration étant : $\mathrm{y}=4,6636 \mathrm{x}-135,89$. Posons $\mathrm{Y}=$ 4,6636Q -135,89 avec Q la quantité en artémisinine correspondant à une surface sous courbe $\mathrm{Y}$. La teneur en artémisinine exprimée en $\%=2 \times 10^{6} \mathrm{Q}$; Q étant exprimée en g. La teneur en artémisinine a été calculée dans la plante après chacun des quatre dosages d'une journée. Les Tableaux 3, 4 et 5 renferment respectivement les teneurs obtenues au cours des première, deuxième et troisième journées. La teneur moyenne en artémisinine dans la plante est: 0,302 \pm $0,008 \%$.

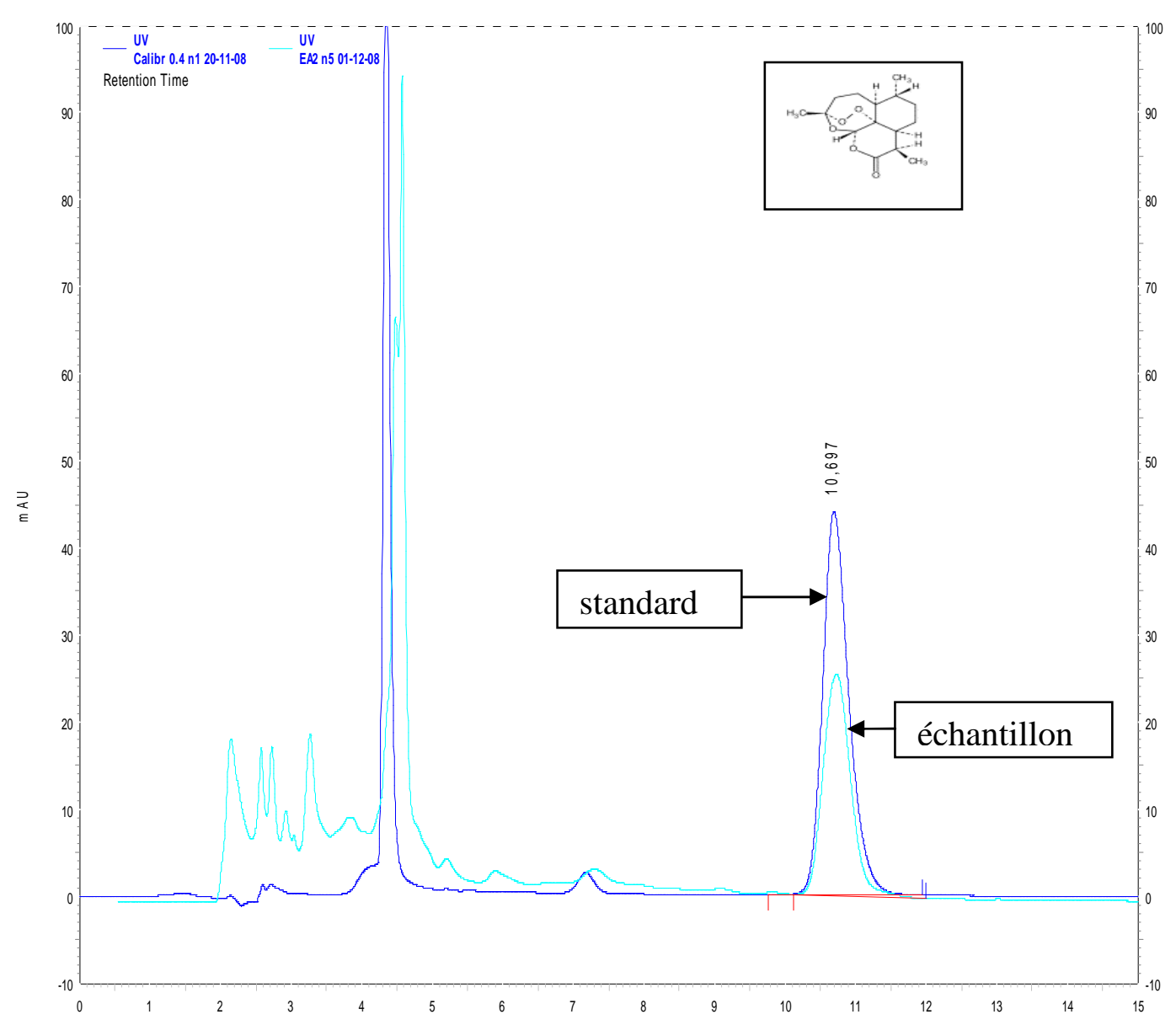

Figure 3: Chromatogrammes comparés du témoin et d'un échantillon. 
Tableau 1: Coefficients de variations(CV) des analyses intra- jour et inter -jours.

\begin{tabular}{ccc}
\hline \multirow{2}{*}{$\begin{array}{l}\text { Standard Quantité } \\
(\mathbf{n g})\end{array}$} & \multicolumn{2}{c}{ CV $(\%)$} \\
\cline { 2 - 3 } & Intra -jour $(\mathbf{n}=\mathbf{3})$ & Inter -jours $(\mathbf{n}=\mathbf{4})$ \\
\hline $\mathbf{2 0 0}$ & $\mathbf{4 , 6 7}$ & $\mathbf{2 , 9 7}$ \\
$\mathbf{4 0 0}$ & $\mathbf{3 , 5 5}$ & $\mathbf{2 , 0 5}$ \\
$\mathbf{8 0 0}$ & $\mathbf{0 , 8 7}$ & $\mathbf{1 , 4 3}$ \\
$\mathbf{1 2 0 0}$ & $\mathbf{2 , 0 2}$ & 3,09 \\
& & \\
\hline
\end{tabular}

Tableau 2: Limite de détection(LD) et Limite de quantification(LQ).

\begin{tabular}{lcc}
\hline \multirow{2}{*}{$\begin{array}{l}\text { Standard } \\
\text { quantité }(n g)\end{array}$} & \multicolumn{2}{c}{ LD et LQ } \\
\cline { 2 - 3 } & LD & LQ \\
\hline 200 & 0,331 & 1,104 \\
400 & 0,485 & 1,617 \\
800 & 0,693 & 2,310 \\
1200 & 2,371 & 7,905 \\
\hline
\end{tabular}

Tableau 3 : Teneur en artémisinine (première journée).

\begin{tabular}{lccc}
\hline Dosage & Y en AUC & $\begin{array}{c}\mathbf{Q}=(\mathbf{Y + 1 3 5 , 8 9}) / 4,6636 \\
\text { en ng }\end{array}$ & $\begin{array}{c}\text { Teneur en } \\
\text { artémisinine } \\
\text { exprimée en \% }\end{array}$ \\
\hline $\mathrm{N}^{\circ} 1$ & 570,415 & 151,4701 & 0,303 \\
$\mathrm{~N}^{\circ} 2$ & 524,504 & 141,6243 & 0,282 \\
$\mathrm{~N}^{\circ} 3$ & 580,699 & 153,656 & 0,307 \\
$\mathrm{~N}^{\circ} 4$ & 525,206 & 141,7748 & 0,283 \\
\hline
\end{tabular}

Tableau 4 : Teneur en artémisinine (deuxième journée).

\begin{tabular}{lccc}
\hline Dosage & $\begin{array}{c}\text { Y en } \\
\text { AUC }\end{array}$ & $\begin{array}{c}\mathbf{Q}=(\mathbf{Y + 1 3 5 , 8 9}) / \mathbf{4 , 6 6 3 6} \\
\text { en ng }\end{array}$ & $\begin{array}{c}\text { Teneur en } \\
\text { artémisinine } \\
\text { exprimée en \% }\end{array}$ \\
\hline $\mathrm{N}^{\circ} 1$ & 598,347 & 157,440 & 0,314 \\
$\mathrm{~N}^{\circ} 2$ & 557,947 & 148,777 & 0,297 \\
$\mathrm{~N}^{\circ} 3$ & 532,436 & 143,3069 & 0,286 \\
$\mathrm{~N}^{\circ} 4$ & 579,286 & 153,353 & 0,306 \\
\hline
\end{tabular}


Tableau 5 : Teneur en artémisinine (troisième journée).

\begin{tabular}{lccc}
\hline Dosage & Y en AUC & $\begin{array}{c}\mathbf{Q}=(\mathbf{Y + 1 3 5 , 8 9}) / \mathbf{4 , 6 6 3 6} \\
\text { en ng }\end{array}$ & $\begin{array}{c}\text { Teneur en artémisinine } \\
\text { exprimée en\% }\end{array}$ \\
\hline $\mathrm{N}^{\circ} 1$ & 558,096 & 148,809 & 0,298 \\
$\mathrm{~N}^{\circ} 2$ & 572,671 & 151,934 & 0,304 \\
$\mathrm{~N}^{\circ} 3$ & 609,911 & 159,920 & 0,320 \\
$\mathrm{~N}^{\circ} 4$ & 614,988 & 161,008 & 0,322 \\
\hline
\end{tabular}

\section{DISCUSSION}

Cette méthode d'extraction utilise un système qui donne une macération répétée avec renouvellement de solvant, mais permet aussi d'obtenir les portions de composés solubles de la plante dans un petit volume de solvant. Le principal risque est la perte des composés thermolabiles.

Les résultats obtenus montrent que la méthode d'extraction (Extraction au Soxhlet) a permis d'extraire la majorité d'artémisinine contenue dans la plante. La littérature rapporte plusieurs procédés d'extraction de l'artémisinine faisant intervenir des solvants différents (Christen and Veuthey, 2001; Lapkin et al., 2006).

La méthode analytique de dosage inspirée de celle de Zhao et Zeng (1985) a conduit à l'homogénéité des deux chromatogrammes: le chromatogramme analysé est bien celui de l'artémisinine. Ces chromatogrammes montrent respectivement les pics correspondants à l'artémisinine dans le standard et dans l'échantillon.

Les coefficients de variations intra-jour (répétabilité) et inter-jours (fidélité intermédiaire) obtenus sont inférieurs aux normes SFSTP, soit 5\% pour la stratégie utilisée. Les teneurs en artémisinine sont néanmoins faibles par rapport à celles de la société Action Nature Médecine (Anamed). Il parait important de noter que la teneur en artémisinine du thé que la société Anamed commercialise varie entre 0,2 et $1 \%$.

\section{Conclusion}

La méthode de chromatographie liquide à haute performance a été développée en utilisant un modèle linéaire pour l'identification, la quantification de l'artémisinine dans la poudre de feuilles de Artemisia аппиа: la teneur moyenne en artémisinine dans la plante est de 0,302 \pm $0,008 \%$ avec un temps de rétention moyen égal à 10,7 min. Les coefficients de variations intra-jour ou répétabilité $(n=3)$ et inter-jours ou fidélité intermédiaire $(\mathrm{n}=4)$ sont inférieurs à $5 \%$ pour toute la série des quantités en artémisinine analysées $(200,400,800$ et 1200 ng). Cette méthode présente une bonne répétabilité et fidélité pour la gamme de quantités en artémisinine analysées. Elle est simple, rapide, précise, reproductible, fiable mais onéreuse. Elle peut être utilisée pour l'analyse quantitative d'autres principes actifs analogues.

\section{REFERENCES}

Bhandari P, Gupta AP, Singh B, Kaul VK. 2005. Simultaeous densitometric determination of artemisinin, artemisinic acid and arteannuin-B in Artemisia апnиа using reversed-phase thin layer chromatography. J. Sep. Sci., 28: 22882292.

Celeghini RMS, Silva AP, Sousa IMO, Foglio MA. 2006. Evaluation of Artemisia аппиа L. clean up methods for artemisinin quantification by HPLC. Rev. Brasil Plant. Med., 8: 119-122.

Christen P, Veuthey JL. 2001. New trends in extraction, identification and quantification of artemisinin and its derivatives. Curr. Med. Chem., 8: 18271839.

Davis TM, Karunajeewa HA, Llett KF. 2005. Artemisinin-based combination therapies 
for uncomplicated malaria. Med. J. Aust., 182(4): 181-185.

Elisabeh H. 2006. The history of qing hao in the Chinese material medica. The Royal Society of Tropical Medicine and Hygiene, 100: 505-508.

European Pharmacopeia. 2004. European Council, Strasbourg. Gabriels M, PlaizierVercammen J. 2004. Development of reversed-phase thin-layer Chromatographic method for artemisinin and its derivatives. J. Chromatogr. Sci., 42: 341347.

Hans MH, Konda KM, Innocent B. 2006. La Médecine Naturelle Tropicale: Traitements. Anamed ; 23-29.

Hu XR, She FH. 2006. Determination of artemisinin content in Artemisia annua from different regions by HPLCevaporative light scattering detection. Xiandai Shipin Yu Yaopin Zazhi, 16: 34 36.

Hubert P. 2000. Commission SFSTP (Société Française des Sciences et Techniques Pharmaceutiques). Validation des Procédures Analytiques Quantitatives: Harmonisation des Démarches, Version 4.3.

Hubert P. 2006.Validation des méthodes analytiques. Université de liège ; 45-46.

Jorge F, Javier MG. 2009. Analysis of underivatized artemisinin and related sesquiterpene lactones by HPLC with ultraviolet detection. Phytochemical Analysis, 20: 91-97.

Lapkin AA, Plucinski PK, Cutler M. 2006. Comparative assessment of technologies for Extraction of Artemisinin. J. Nat. Prod., 69: 1653-1664.

Marchand E, Atemnkeng, Magnus A, Vanermen, Stijn. 2008. Development and validation of a simple thin layer chromatographic method for the analysis of artemisinin in Artemisia annua L. plant extracts. Biomedical Chromatography, 22(5): 454-459.

Marchese JA, Rehder VLG, Sartoratto A. 2001. Quantification of artemisinin in Artemisia annua L. A comparison of thinlayer Chromatography with densitometric detection and high performance liquid chromatography with UV detection. Rev. Brasil Plant. Med., 4: 81-87.

Myriam G, Simmonet X. 2002. Dosage de l'artémisinine par Chromatographie sur Couche Mince(CCM). Revue Suisse de Vitic. Arboric. Hortic, 34(3): 205-208.

Peng CA, Ferreira JFS, Wood AJ. 2006. Direct analysis of artemisinin from Artemisia annua L. using highperformance liquid chromatography with evaporative light scattering detection, and gas chromatography with flame ionization detector. Journal of Chromatography A., 1133: 254-258.

Qian GP, Yang YW, Ren QL. 2005. Determination of artemisinin in Artemisia annua L. by reversed phase HPLC. J. Liq. Chrom. \& Rel. Technol., 28: 705-712.

Rodger WS, Kenneth GL, Peter M, Greg K, István P, Christina K. 2009. High performance liquid chromatographic evaluation of artemisinin, raw material in the synthesis of artesunate and artemether. Journal of Chromatography A., 1216: 8918-8925.

Towie N. 2006. Malaria breakthrough raises spectre of drug resistance. Nature, 440(7086): 852-853.

Zhao SS, Zeng MY. 1985. Spekmetrische Hochdruck-Chromatograishe (HPLC) Untersuchungen zur Analytik von Qinghaosu. Planta Med., 51: 233-237. 